\title{
Solitary intramuscular cysticercosis involving the triceps muscle a case Report
}

\section{Chennamaneni V}

Dr Vikas Chennamaneni,Assistant Professor in Radiodiagnosis, Prathima Institute of Medical Sciences, Nagunoor, Karimnagar dist, Andhra Pradesh, India.

Address for correspondence: Dr Vikas Chennamaneni, E mail: vikas_ch_rao@yahoo.com

\begin{abstract}
Intramuscular cysticercosis is a less common presentation and high resolution ultrasound can help in clinching the diagnosis and avoiding unnecessary interventions. Patient can be managed conservatively with antihelminths and steroids. We present the case of a 23-year-old man with a swellingon theposterior side of right arm and was diagnosed as intramuscularcysticercosis in the triceps muscle on high-resolution ultrasound and managed conservatively with antihelminths.
\end{abstract}

Key-words: Intramuscular cysticercosis, Triceps muscle, High resolution ultrasound

\section{Introduction}

Cysticercosis in humans is infection by the larval form (cysticercus cellulosae) of the pork tapeworm Taenia solium. The location of cysts in order of frequency is the central nervous system, subcutaneous tissue and striated muscle, vitreous humour of the eye and, rarely, other tissues. Intramuscular cysticercosis is a less common presentation and high resolution ultrasound can help in clinching the diagnosis and avoid unnecessary interventions. We describe a rare case of cysticerosis in the triceps muscle, diagnosed only with ultrasound and managed conservatively. Most cases with muscular involvement present with multiple cysts. Central nervous system involvement is also common. Our case is a rare and unusual manifestation of cysticercosis presenting as an isolated swelling of triceps muscle without involvement of central nervous system or other organ system, and discovered in a person who never consumed pork.

Manuscript received: $16^{\text {th }}$ July 2013

Reviewed: $26^{\text {th }}$ July 2013

Author Corrected: $29^{\text {th }}$ July 2013

Accepted for Publication: 30 $30^{\text {th }}$ July 2013
In electronic databases not more than 10 cases have been reported of Muscular cysticercosis ${ }^{1-6}$.

\section{Case Report}

A 23 -year-old man reported to the Department of Radiology and imaging, of Tertiary care teaching Unit in South India, with a swelling on posterior aspect of right arm of two months duration. The swelling was gradually increasing in size. On examination, there was a large swelling of approximately $4 \times 3 \mathrm{~cm}$ on the posterior aspect of right arm, in the middle third. The swelling was firm, tender andnon-fluctuant, with stretched overlying skin and increased temperature suggestive of inflammatory pathology. Systemic examination of the patient was normal and there was no other swelling.

The patient was residing in a area where sanitation conditions were poor and although he never consumed pork. In the haematological investigations, there was a mild eosinophilia. Other haematological finding and chest 
radiograph were normal. The patient was sent to the Department of Radiology and Imaging for an ultrasound examination of the swelling. Ultrasound was performed on a PHILIPS HD 7 machine with a linear probe at $10 \mathrm{MHz}$ frequency. On ultrasound there was a well-defined cystic lesion measuring $2.6 \times 2.3 \mathrm{~cm}$ and containing a small echogenic nidus (Figure 1), and there was a large

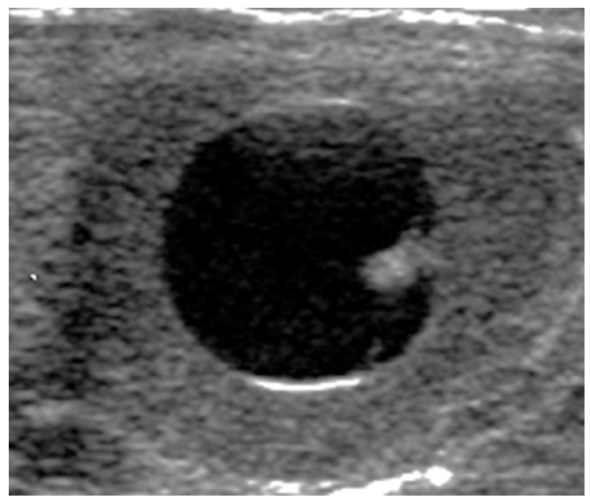

Fig 1: well-defined cystic lesion containing a small echogenic nidus

hypoechoic area $3.5 \times 4.0 \mathrm{~cm}$ around the cystic lesion in the right triceps. Therefore, on ultrasound examination, a diagnosis of cysticercosis in the right triceps muscle with surroundinginflammatory phlegmon was made.

The patient was managed conservatively and prescribed albendazole $400 \mathrm{mg}$ tablets twice daily for 30 days along with tapered dose of steroids. After 30 days of conservative treatment, the lesion showed regression.

\section{Discussion}

Cysticercosis is an infection with the larval stage of Taenia solium. It is seen as cysts in various human tissues, more commonly in the brain and the orbit. It is endemic in most parts of Asia and is transmittedby the fecal-oral route ${ }^{7}$.

Tapeworm infection is common in developing countries where thecombination of rural society, crowding, and poor sanitationallows greater contact between humans and pigs and thus more opportunities for fecal contamination of food and water.
Humans normally act as definitive hosts. Ingestion of inadequately cooked infected pork, the intermediate host, leads to the development of the adult worm in the small bowel of humans.

The eggs of the worm are excreted with the feces, which are ingested by the pig, the intermediate host. Once ingested, the eggs hatch in the small intestine and result in the cysticercosis, completing the cycle. However, humans can occasionally be intermediate hosts, manifesting cysticercosis.

It is transmitted to humans by ingestion of eggs from contaminated water or food, such as vegetables,or by internal regurgitation of eggs into the stomach due to reverse peristalsis, when the intestine harbors a gravid worm $^{8}$. The eggs hatch in the small intestine releasing oncospheres that penetrate the bowel mucosa and enter the bloodstream to reach various tissues, where they develop to form a cysticercus cellulosae, which is the encysted larval form of Taenia Solium. These can remain viable in this stage for as long as 10 years in humans.

Living larvae evade immune recognition and do not elicit inflammation. When the larva dies, it induces a vigorous granulomatous inflammatory response that may produce symptoms, depending on the anatomic location ${ }^{9}$.

Cysticercosis is the most common parasitic disease of the

central nervous system worldwide, but intramuscular cysticercosis has been reported much less frequ ntly. Cutaneous parasitism by larval cestodes can take the form of subcutaneous nodules, generating a clinical differential diagnosis of infundibular cyst, lipoma, neurofibroma, reactive lymph nodes, granular cell tumor as well as malignant tumors ${ }^{10-11}$.

The muscular form of cysticercosis, when confined to muscles, is generally asymptomatic, although three distinct types of clinical manifestations have been described: the myalgic, myopathic type; the nodular or masslike type; and the rare pseudohypertrophytype ${ }^{12}$ 
During the death of the larva, there is leakage of fluid from the cyst. The resulting acute inflammation may result in local pain and myalgia. Alternatively, degeneration of the cyst may result in intermittent leakage of fluid, eliciting a chronic inflammatory response, with collection of fluid around the cyst, resulting in the mass-like type, the pseudotumour type or the abscess-like type, as was seen in our case. Alternatively, the cyst retracts, its capsule thickens and the scolex calcifies.

Four different sonographic appearances of muscular cysticercosis have been described ${ }^{13}$. These appearances on high-resolution sonography are pathognomonic of cysticercosis, and a definitive diagnosis can be made with greater confidence. One of the sonographic appearances of cysticercosis is the cysticercus cyst with an inflammatory mass around it, which occurs as a result of the death of the larva.

The second type is an irregular cyst with very minimal fluid on one side, indicating a leakage of fluid. The eccentric echogenic protrusion from the wall caused by the scolex is not seen withinthe cyst. It may be due to escape of the scolex to outside thecyst or partial collapse of the cyst. The third appearance is a large

irregular collection of exudative fluid within the muscle with the typical cysticercus cyst containing a scolex situatede ccentrically within the collection. This may be due to chronic intermittent leakage of fluid from the cyst, leading to florid inflammatory exudates. This appearance is similar to an intramuscular abscess, but visualization of the cysticercus cyst within it clinches the diagnosis ${ }^{5}$.

The fourth sonographic appearance is that of calcified cysticercosis. It appears as multiple elliptical calcifications in the soft tissues similar to the pathognomonic millet seed-shaped elliptical calcifications in soft tissuesdescribed on plain radiography. In the first 3 types, the salient diagnostic feature is the cysticercus itself, which appearsas an oval or round well-defined cystic lesion with an eccentric echogenic scolex in it. This feature was shown very well in our case, which had lesions of the third type ${ }^{6}$.

In conclusion, high-resolution ultrasound, being noninvasiveand non-ionizing, plays an important role in establishing thediagnosis in patients with muscular cysticercosis.

If lesionswith the morphological characteristics described above are encounteredon ultrasound, the diagnosis of cysticercosis can be made withgreat confidence, and in muscular cysticercosisno further investigation is required and the patient can be managed conservatively. The prerequisite of successful treatment with anti-helminthics is early diagnosis.Medical treatment with praziquantel or albendazolehas been recommended for neurocysticercosis and subcutaneous cysticercosis ${ }^{4}$.

We have successfully managed the patient conservatively with albendazole and steroids only. Therefore, with the help of non-invasive high-resolution ultrasound examination such subcutaneous and intramuscular cysticerci can be accurately diagnosed without the need of invasive biopsy or fine needle aspiration cytology (FNAC).

\section{References}

1. Sethi PK, Sethi NK, Torgovnick J, Arsura E. Cysticercosis of temporalis muscle: an unusual cause of temporal headaches. A case report. J Headache Pain. 2007 Oct; $8(5): 315-6$

\section{Singal R, Mittal A, Gupta S, Gupta R, Sahu P, Gupta A.} Intramuscular cysticercosis diagnosed on ultrasonography in thigh: A rare case report. N Am J Med Sci. 2010 Mar;2(3):162-4

3. Ergen FB, Turkbey B, Kerimoglu U, Karaman K, Yorganc K, Saglam A. Solitary cysticercosis in the intermuscular area of the thigh: a rare and unusual pseudotumor with characteristic imaging findings. $\mathrm{J}$ Comput Assist Tomogr. 2005 Mar-Apr;29(2):260-3 
4. Schmidt DK, Jordaan HF, Schneider JW , Cilliers J. Cerebral and subcutaneous cysticercosis treated with albendazole. Int J Dermatol 1995;34:574-9.

5. Mani NB, Kalra N, Jain M, Sidhu R. Sonographic diagnosis of a solitary intramuscular cysticercal cyst. J Clin Ultrasound 2001;29:472-475.

6. Ogilvie CM, Kasten P, Rovinsky D, Workman KL, Johnston JO. Cysticercosis of the triceps - an unusual pseudotumor: case report and review. Clin Orthop 2001; $382: 217-221$

7. Despommier DD. Tapeworm infection: the long and the short of it. N Engl J Med 1992; 327:727-728.

8. Abdelwahab IF, Klein MJ, Hermann G, Abdul-Quader M. Solitary cysticercosis of the biceps brachii in a vegetarian: a rare and unusual pseudotumor. Skeletal Radiol 2003; 32:424-428.
9. Botero D, Tanowitz HB, Weiss LM, Wittner M. Taeniasis and cysticercosis. Infect Dis Clin North Am $1993 ; 7: 683-697$

10. Singrodia S, Joshi RG, Solanki RB, Rawal RC. Subcutaneous nodules preceding convulsions due to neural cysticercosis. Indian J Dermatol Venereol Leprol 2008;74:385-6.

11. Mittal A, Gupta S, Gupta S, Mehta V. Subcutaneous and intramuscular cysticercosis: High- resolution sonography. Indian J Dermatol Venereol Leprol 2009;75:515-6

12. Mittal A, Das D, Iyer N, Nagaraj J, Gupta M. Masseter cysticercosis- a rare case diagnosed on sonography. Dentomaxillofac Radiol 2008;37:113-6.

13. Vijayaraghavan SB. Sonographic appearances in cysticercosis. J Ultrasound Med 2004;23:423-7

\section{How to cite this article?}

Chennamaneni V. Solitary intramuscular cysticercosis involving the triceps muscle a case Report. Int J Med Res Rev 2013;1(3):142-145. doi: 10.17511/ijmrr.2013.i03.12 